\title{
Thermal Emission in the Southwest Clump of VY CMa ${ }^{*}$
}

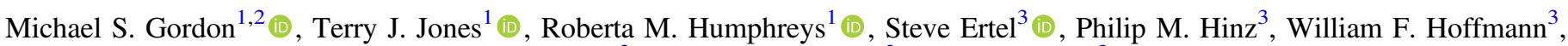 \\ Jordan Stone $^{3}$ (D) , Eckhart Spalding ${ }^{3}$, and Amali Vaz ${ }^{3}$ \\ ${ }^{1}$ Minnesota Institute for Astrophysics, School of Physics and Astronomy, University of Minnesota, 116 Church Street SE, Minneapolis, MN 55455, USA \\ gordon@astro.umn.edu \\ ${ }^{2}$ SOFIA Science Center, NASA Ames Research Center, Moffett Field, CA 94035, USA \\ ${ }^{3}$ Steward Observatory, Department of Astronomy, University of Arizona, 993 N. Cherry Avenue, Tucson, AZ 85721, USA \\ Received 2018 May 21; revised 2018 November 28; accepted 2018 December 2; published 2019 January 18
}

\begin{abstract}
We present high spatial resolution LBTI/NOMIC 9-12 $\mu \mathrm{m}$ images of VY CMa and its massive outflow feature, the Southwest (SW) Clump. Combined with high-resolution imaging from the Hubble Space Telescope $(0.4-1 \mu \mathrm{m})$ and LBT/LMIRCam $(1-5 \mu \mathrm{m})$, we isolate the spectral energy distribution (SED) of the clump from the star itself. Using radiative-transfer code DUSTY, we model both the scattered light from VY CMa and the thermal emission from the dust in the clump to estimate the optical depth, mass, and temperature of the SW Clump. The SW Clump is optically thick at $8.9 \mu \mathrm{m}$ with a brightness temperature of $\sim 200 \mathrm{~K}$. With a dust chemistry of equal parts silicates and metallic iron, as well as assumptions on grain size distribution, we estimate a dust mass of $5.4 \times 10^{-5} M_{\odot}$. For a gas-to-dust ratio of 100 , this implies a total mass of $5.4 \times 10^{-3} M_{\odot}$. Compared to the typical mass-loss rate of VY CMa, the SW Clump represents an extreme, localized mass-loss event from $\lesssim 300$ yr ago.
\end{abstract}

Key words: stars: individual (VY CMa) - stars: mass-loss - stars: winds, outflows - supergiants

\section{Introduction}

The extreme red supergiant VY Canis Majoris is one of the brightest infrared sources in the sky. Hubble Space Telescope (HST) imaging and long-slit spectroscopy from 0.4 to $1 \mu \mathrm{m}$ reveal a complex circumstellar nebula environment with multiple arcs and knots (Smith et al. 2001; Humphreys et al. $2005,2007)$. Ejected in separate mass-loss events over the past $\sim 1000 \mathrm{yr}$, these features are structurally and kinematically distinct from the surrounding nebulosity.

Shenoy et al. (2013) extended the exploration of VY CMa's ejecta into the near- to mid-infrared with higher spatial resolution than previous studies with ground-based $1-5 \mu \mathrm{m}$, adaptive optics imaging using LMIRCam (Skrutskie et al. 2010) on the Large Binocular Telescope (LBT). The dominant IR source in the $2.2,3.8$, and $4.8 \mu \mathrm{m}\left(K_{s^{-}}, L^{\prime}-\right.$, and $M$-band) images is the peculiar "Southwest Clump" (hereafter, SW Clump), which is optically thick in the HST/WFPC2 images at $1 \mu \mathrm{m}$ (Smith et al. 2001). Shenoy et al. (2013) determined that the high surface brightness of the SW Clump requires optically thick scattering at wavelengths shorter than $5 \mu \mathrm{m}$, rather than thermal emission from dust grains because the expected blackbody equilibrium temperature for material $\sim 1500$ au from the central star is quite low ( $\lesssim 170 \mathrm{~K})$.

Scattering as the dominant component of the SW Clump has been confirmed using high-resolution imaging polarimetry in the near-IR. Using MMT-Pol (Packham et al. 2012) on the $6.5 \mathrm{~m}$ MMT Observatory at Mt. Hopkins, Shenoy et al. (2015) observed $\sim 30 \%$ fractional polarization in the clump at $3.1 \mu \mathrm{m}$, which requires optically thick scattering from low albedo dust grains. In

\footnotetext{
* The LBT is an international collaboration among institutions in the United States, Italy, and Germany. LBT Corporation partners are The University of Arizona on behalf of the Arizona Board of Regents; Istituto Nazionale di Astrofisica, Italy; LBT Beteiligungsgesellschaft, Germany, representing the Max-Planck Society, The Leibniz Institute for Astrophysics Potsdam, and Heidelberg University; The Ohio State University, and The Research Corporation, on behalf of The University of Notre Dame, University of Minnesota, and University of Virginia.
}

earlier work, Shenoy et al. (2013) estimate a lower limit on the total mass within the clump of $0.5-2.5 \times 10^{-2} M_{\odot}$ depending on the assumed gas-to-dust ratio (see the discussion in Section 3.4). In any case, this ejecta event can be contrasted with VY CMa's "normal" mass-loss rate of $\sim 10^{-4} M_{\odot} \mathrm{yr}^{-1}$ (Danchi et al. 1994; Humphreys et al. 2005; Decin et al. 2006), suggesting that the SW Clump represents a single mass-loss episode from a localized region of VY CMa's stellar atmosphere.

Recent submillimeter observations with ALMA reveal dusty concentrations within $\sim 10 R_{\star}$ of VY CMa (Richards et al. 2014; O'Gorman et al. 2015; Vlemmings et al. 2017), adopting the Wittkowski et al. (2012) measurement $R_{\star}=1420 R_{\odot}$. O'Gorman et al. (2015) found a cold clump to the southeast, "Clump C," located closer to VY CMa than the SW Clump-400 au $\left(61 R_{\star}\right)$ versus 1500 au $\left(230 R_{\star}\right)$. While O'Gorman et al. (2015) estimate a dust mass lower limit of $2.5 \times 10^{-4} M_{\odot}$ for Clump C, similar to the SW Clump, there is no evidence for the SW Clump in the ALMA images at 321 and $658 \mathrm{GHz}$ (Bands 7 and 9; O'Gorman et al. 2015) or at $178 \mathrm{GHz}$ (Band 5; Vlemmings et al. 2017) in thermal emission. Kamiński et al. (2013) did not observe the SW Clump in thermal emission with the Submillimeter Array (SMA), though it was observed in line maps of $\mathrm{H}_{2} \mathrm{~S}(300.5 \mathrm{GHz})$, CS $(293.9 \mathrm{GHz})$, and in several other molecular transitions. Given the mass estimates of the SW Clump from the LMIRCam and MMT-Pol observations in Shenoy et al. (2013, 2015), the nondetection in thermal emission in the ALMA bands may have implications for the dust grain properties in the far-IR.

Even without detection of continuum emission of the SW Clump in the radio, molecular transition studies from ALMA and the SMA are useful in tracing the geometry of the clump, particularly the clump's orientation relative to the plane of the sky. Slightly blueshifted $\mathrm{TiO}_{2}$ emission observed in ALMA observations (De Beck et al. 2015) appears coincident with scattered light in the $1 \mu \mathrm{m} H S T$ images (Smith et al. 2001) and suggests that the SW Clump-or at least material between the clump and the star-is partially in front of the plane of the sky (De Beck et al. 2015). However, $\mathrm{NaCl}$ emission at the 


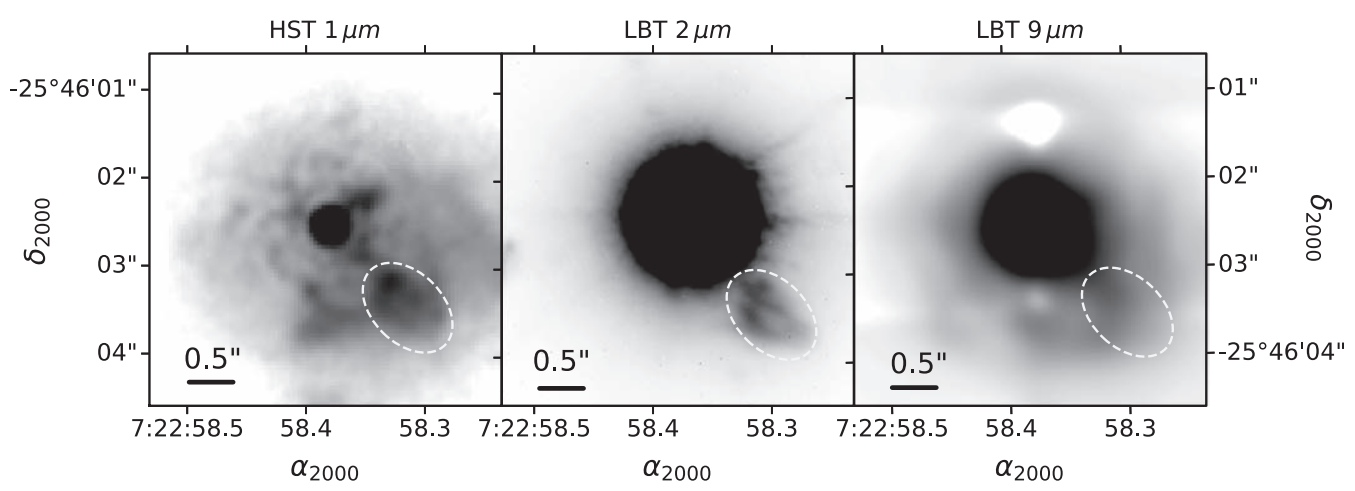

Figure 1. Left: $H S T$ /WPFC2 $1 \mu \mathrm{m}$ (F1042M; Smith et al. 2001). Center: LBT/LMIRCam $2.2 \mu \mathrm{m}$ ( $K_{s}$-band; Shenoy et al. 2015). Right: LBT/NOMIC 8.9 $\mu \mathrm{m}$ (this work). The white bands in the NOMIC image are artifacts due to column saturation around the central star. The white dashed region represents the elliptical aperture from Shenoy et al. (2013) defined as roughly $1 \sigma$ above the background in the LMIRCam $K_{s}$ image. This region is $\sim 0.6 \times 0$.! 4 , centered $\sim 1{ }^{\prime \prime} 5$ from the star, and inclined $45^{\circ}$ east from north.

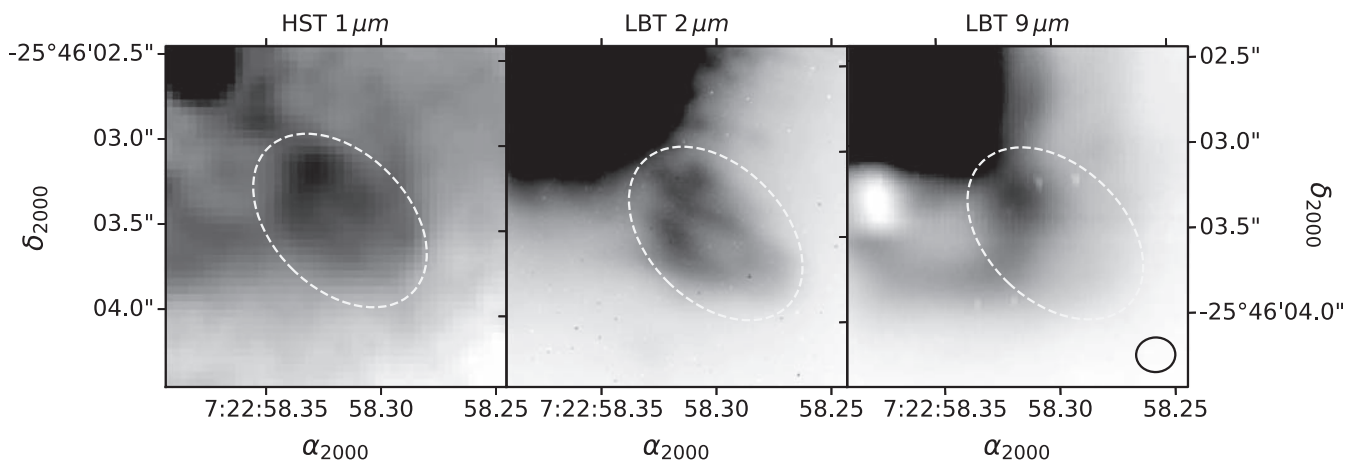

Figure 2. Same as in Figure 1 but zoomed in on the SW Clump feature. The black ellipse in the bottom right corner is the FWHM of the PSF of the NOMIC $8.9 \mu \mathrm{m}$ image.

location of the SW Clump appears redshifted at $\sim 3 \mathrm{~km} \mathrm{~s}^{-1}$ (Decin et al. 2016) with respect to the LSR velocity, consistent with the Humphreys et al. (2007) kinematic study of HST images. For the analysis in this work, we assume, then, that the SW Clump is at least close to the plane of the sky.

In this study, we present LBT/Nulling Optimized MidInfrared Camera (NOMIC) (Hoffmann et al. 2014) 8.9, 10.3, and $11.9 \mu \mathrm{m}$ imaging and photometry of VY CMa and its SW Clump. While the earlier LMIRCam observations reveal the scattered light of the dusty clump, NOMIC imaging provides measurements of the thermal emission of the dusty grains. We model the spectral energy distributions of both VY CMa and the SW Clump separately using the radiativetransfer code DUSTY (Ivezic et al. 1997) to show that the thermal emission at $8-12 \mu \mathrm{m}$ is largely consistent with a nondetection by ALMA at $400-1000 \mu \mathrm{m}$, but slightly above the ALMA detection limit at $1.7 \mathrm{~mm}$.

\section{Observations and Data Reduction}

We observed VY CMa with NOMIC on UT 2017 January 12 with a single $8.4 \mathrm{~m}$ primary mirror on the LBT. The NOMIC (Hoffmann et al. 2014) is part of the Large Binocular Telescope Interferometer (LBTI; Hinz et al. 2016) system. It uses a $1024 \times 1024$ Si:As array with a pixel scale of 0 ". $018 \mathrm{pix}^{-1}$ and provides a field of view of $12^{\prime \prime} \times 12^{\prime \prime}$. Images were made at $8.9 \mu \mathrm{m}(\Delta \lambda=0.76 \mu \mathrm{m}), 10.3 \mu \mathrm{m}$ $(\Delta \lambda=6.0 \mu \mathrm{m})$, and $11.9 \mu \mathrm{m}(\Delta \lambda=1.13 \mu \mathrm{m})$ with individual exposure times of $27.5 \mathrm{~ms}$ for a total of $\sim 90 \mathrm{~s}$ in each filter ( 3200 individual frames). The exposure times were short to mitigate saturation from the central star, and the telescope was nodded between two positions on the NOMIC chip to ease background subtraction in data reduction. The reduced $8.9 \mu \mathrm{m}$ image is shown in Figure 1 on the right, aligned with the HST/ WFPC2 $1 \mu \mathrm{m}$ image from Smith et al. (2001) and the LBT/ LMIRCam $K_{s}$-band image from Shenoy et al. (2015). Figure 2 shows the same three frames zoomed in on the SW Clump, with the three observed NOMIC bands (8.9, 10.3, and 11.9 microns) shown in Figure 3.

Sirius was observed at similar airmass and with the same nod locations on the NOMIC array for both flux and point-spread function (PSF) calibration. The PSFs were modeled in each wavelength at each nod position using the Astropy (Astropy Collaboration et al. 2013) fitting functions for a twodimensional Gaussian. For flux calibration, we used photometry of Sirius with Gemini/T-ReCS from Skemer \& Close (2011). The T-ReCS and NOMIC filters have similar central wavelengths but different filter bandwidths, so we scale our measured counts into "synthetic filters" to effectively interpolate the Sirius photometry into the NOMIC filter sets. This filter correction permits flux calibration of our VY CMa images.

For each nod position, the $\sim 3200$ frames in each filter are mean-combined with a sigma clipping threshold of three standard deviations from the average in each pixel. The two nod position images are subtracted from each other, VY CMa is masked out, and the background rms in each NOMIC 


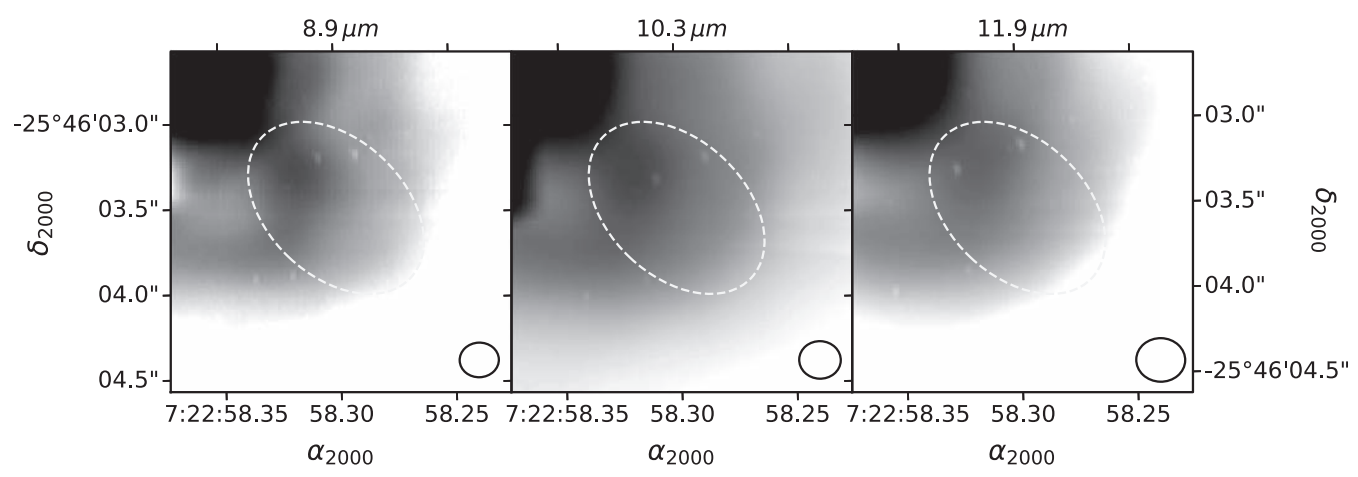

Figure 3. Observations in all three NOMIC filters zoomed of the SW Clump feature. The observed PSF in each band is indicated in the bottom right of each panel. We have tried to scale these images to clearly display the clump, but the brightness of VY CMa creates contrast issues.

amplifier is modeled separately using the Astropy-affiliated photutils ${ }^{4}$ package.

\section{Results and Discussion}

\subsection{Photometry of the SW Clump}

To quantify the flux in the SW Clump relative to VY CMa's SED, we need to subtract the contribution from the central star itself. In a manner similar to Shenoy et al. (2013) we scale the amplitude of the PSF models from the Sirius images to match the profile of VY CMa. Since the central star is partly saturated, the "wings" of the PSF are used in the scaling to both locate the centroid and scale the amplitude. While centroiding on a saturated source can be uncertain, at the distance of the SW Clump from the star, the flux contribution from the PSF was minimal $(\lesssim 10 \%$ of the flux in the clump at $8.9 \mu \mathrm{m})$. In all three bands, saturation from the central star causes column bleed artifacts, but this saturation fortunately missed our aperture of interest.

We recalculate the SW Clump photometry from Shenoy et al. (2013) on the LMIRCam $K_{s^{-}}$, $L^{\prime}-$, and $M$-band images for consistent treatment with the NOMIC images. We generate an aperture around the SW Clump using the $K_{s}$ image to define a region that extends to $1 \sigma$ above the background. This aperture is roughly elliptical $(0.61 \times 0$ !" 44 beam $)$ and centered $\sim 1$ "! 5 from the central star inclined at $45^{\circ}$ east from north. Photometry is performed with photutils, and the same aperture is used in all the LMIRCam and NOMIC images. Additionally, we apply this aperture to the HST/WFPC2, PSFdeconvolved images from Smith et al. (2001) in the F410M, F547M, and F1042M medium-width continuum filters, and the narrow $\mathrm{H} \alpha$ filter $(\mathrm{F} 656 \mathrm{~N})$. In the HST optical images, several arcs, knots, and clumpy features are resolved within the large aperture, so the measured photometry is likely an overestimate. Additionally, without radial velocity measurements of each of these resolved subclumps, we cannot determine which of these features are actually coincident with the SW Clump mass-loss event. However, the aperture photometry in the optical is performed in the same manner as for the IR images for consistency. The SED models described in Section 3.2 do not weight the optical photometry to determine the best fit.

The photometry is summarized in Table 1 . Since the SW Clump is diffuse and we are uncertain of its total spatial extent, we generate a grid of apertures, all with the same total

\footnotetext{
4 photutils provides tools for detecting and measuring the photometry of astronomical sources. The software is still in development, with documentation available at https://photutils.readthedocs.io/.
}

area, but allowing the center to move 0 " 1 in all directions. The error value in Table 1 is the standard deviation of this aperture grid and represents here our measure of systematic uncertainty in the flux. Also included are flux limits for three ALMA bands. VY CMa was observed as ALMA Science Verification data on UT 2013 August 16-19 (321, $658 \mathrm{GHz}$; Richards et al. 2014; O'Gorman et al. 2015) and on UT 2016 October 16 (178 GHz; Vlemmings et al. 2017). As the continuum emission from the SW Clump was undetected in these bands, we instead report a flux limit as $3 \times$ the root mean square (rms) noise in each image, where the measured rms in the ALMA images is scaled to the beam size of our photometric aperture. For example, with the synthesized ALMA beam at $178 \mathrm{GHz}$ of $\sim 0.5 \times 0 .{ }^{\prime \prime} 2$ with an $\mathrm{rms}$ noise of $0.1 \mathrm{mJy}^{-1}$ beam $^{-1}$ (Vlemmings et al. 2017) and our $0.61 \times 0 . " 44$ aperture beam $(2.7 \times$ ALMA beam size), then the detection limit assuming the total flux of the SW Clump is distributed evenly over the beam would be $0.1 \mathrm{mJy}^{\text {beam }}{ }^{-1} \times 2.7$ beams $\times 3$ limit $\approx 0.8$ mJy. These limits are included in Table 1.

The observed SEDs of both VY CMa and the SW Clump are shown in Figure 4. The closed circles represent photometry of VY CMa compiled from the literature, including the $H S T$ / WFPC2 observations at $0.4-1 \mu \mathrm{m}$ plus the ESO $3.6 \mathrm{~m}$ telescope 1-20 $\mu \mathrm{m}$ IR photometry from Smith et al. (2001), and the $20-40 \mu \mathrm{m}$ SOFIA/FORCAST and $60-150 \mu \mathrm{m}$ Herschel/PACS photometry from Shenoy et al. (2016). ${ }^{5}$ The open circles are the extinction-corrected optical and near-IR photometry for foreground (interstellar) $A_{V}=1.5$ (Shenoy et al. 2015) and a traditional extinction curve (Cardelli et al. 1989). The black squares are the photometry from this work on the SW Clump using the elliptical aperture region discussed above in the WFPC2, LMIRCam, and NOMIC images. The $3 \sigma$ ALMA detection limits are shown as downward arrows in the submillimeter to millimeter. The model SED for the clump is fainter than the ALMA limits in the submillimeter regime but slightly above longward of $1 \mathrm{~mm}$, not inconsistent with their nondetection. Nonetheless, our model does put strong constraints on the ALMA results. O'Gorman et al. (2015) suggest that dust properties are different at millimeter waves from those we used to model the mid-infrared wavelength regime.

\subsection{DUSTY Modeling}

To study the thermal properties of the SW Clump, we model the SEDs of both VY CMa and the SW Clump using the

\footnotetext{
5 PACS data obtained as part of the guaranteed time Mass-loss of Evolved StarS (MESS) key program (Groenewegen et al. 2011).
} 
Table 1

Photometry of the SW Clump

\begin{tabular}{|c|c|c|c|c|c|c|}
\hline Telescope & Instrument & $\begin{array}{l}\text { Date Obs. } \\
\text { (UT) }\end{array}$ & Filter & $\begin{array}{c}\lambda_{0} \\
(\mu \mathrm{m})\end{array}$ & $\begin{array}{c}\text { Flux } \\
(\mathrm{Jy})\end{array}$ & $\begin{array}{l}\text { Sys. Error } \\
\text { (Jy) }\end{array}$ \\
\hline$\overline{H S T}$ & WFPC2 & 1999 Mar 22 & F410M & 0.4 & $6.6 \times 10^{-3}$ & $1.9 \times 10^{-3}$ \\
\hline$H S T$ & WFPC2 & 1999 Mar 22 & F547M & 0.5 & 0.1 & 0.04 \\
\hline$H S T$ & WFPC2 & 1999 Mar 22 & F656N & 0.7 & 0.4 & 0.15 \\
\hline$H S T$ & WFPC2 & 1999 Mar 22 & F1042M & 1.0 & 2.1 & 0.91 \\
\hline LBT & LMIRCam & 2011 Nov 16 & $K_{s}$ & 2.2 & 6.8 & 1.2 \\
\hline LBT & LMIRCam & 2011 Nov 16 & $L^{\prime}$ & 3.8 & 15.0 & 4.1 \\
\hline LBT & LMIRCam & 2011 Nov 16 & $M$ & 4.8 & 29.1 & 11.8 \\
\hline LBT & NOMIC & 2017 Jan 12 & 8.9 & 8.9 & 186.9 & 32.4 \\
\hline LBT & NOMIC & 2017 Jan 12 & 10.3 & 10.3 & 318.8 & 75.5 \\
\hline LBT & NOMIC & 2017 Jan 12 & 11.9 & 11.9 & 389.4 & 84.2 \\
\hline ALMA & $\ldots$ & 2013 Aug 16 & Band 9 & 456 & $0.75^{\mathrm{a}}$ & $\ldots$ \\
\hline ALMA & $\ldots$ & 2013 Aug 16 & Band 7 & 934 & $1.6 \times 10^{-2 \mathrm{a}}$ & $\ldots$ \\
\hline ALMA & $\ldots$ & 2016 Oct 16 & Band 5 & 1680 & $8.1 \times 10^{-4 a}$ & $\ldots$ \\
\hline
\end{tabular}

Notes.

${ }^{a}$ Fluxes represent the $3 \sigma$ upper limits estimated from the rms noise in ALMA data scaled to the SW Clump aperture size.

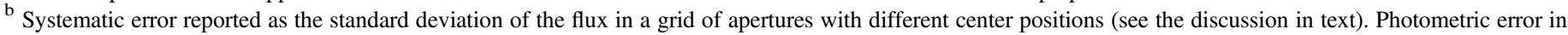
the flux-calibrated NOMIC images is estimated at $<10 \%$.

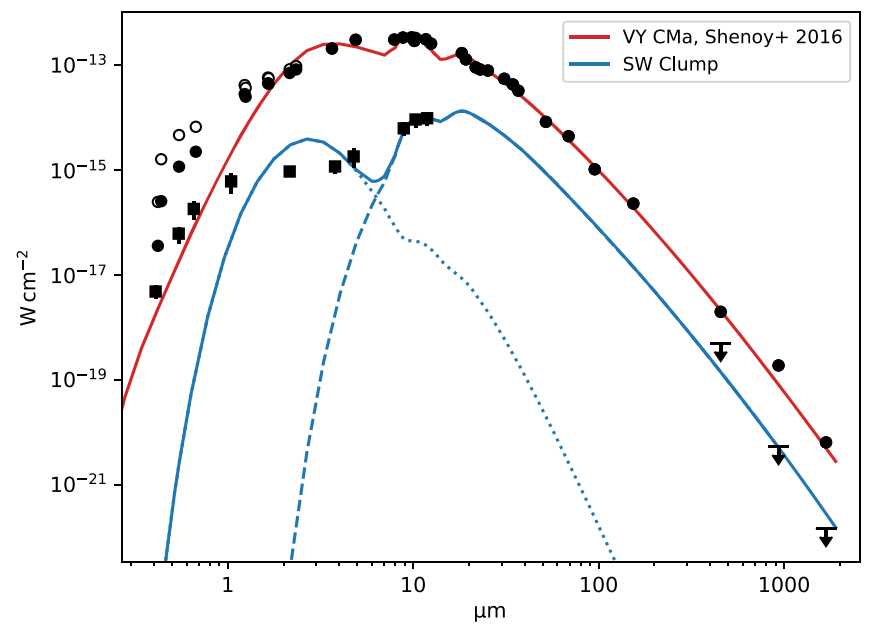

Figure 4. Photometry of VY CMa and the SW Clump. Closed circles represent photometry of VY CMa compiled from the literature. Open circles are the interstellar extinction-corrected optical and near-IR photometry. Black squares are the photometry from this work on the SW Clump from WFPC2 $(0.4-1 \mu \mathrm{m})$, LMIRCam $(2-5 \mu \mathrm{m})$, and NOMIC $(9-12 \mu \mathrm{m})$. The $3 \sigma$ ALMA detection limits are shown as downward arrows in the submillimeter to millimeter. Errorbars represent total uncertainty in the flux calibration, PSFsubtraction, and systematic error in the aperture. The red line represents the best-fitting DUSTY model from Shenoy et al. (2016) using a spherical dust distribution with a density profile of $\rho(r) \propto r^{-1.5}$. The blue line is the best-fit slab model (this work) to the SW Clump. The dotted line is the scattered light component for the slab model, and the dashed line indicates thermal emission, which begins to dominate at $\sim 5 \mu \mathrm{m}$ to longer wavelengths.

DUSTY radiative-transfer code (Ivezic et al. 1997). DUSTY solves the one-dimensional radiative-transfer equation for either a spherically symmetric dust distribution around a central source or through a slab of dusty material. For modeling the SED of VY CMa itself, we employ the spherical mode of DUSTY following previous work in Shenoy et al. (2016), which analyzed the mass-loss histories around hypergiant stars $\mu$ Cep, IRC +10420, $\rho$ Cas, and VY CMa. Shenoy et al. (2016) fit a variety of dust density distributions to each star, and they found that for VY CMa, a density profile of $\rho(r) \propto r^{-1.5}$ best explained the mid-infrared emission in the star's SED.
For our spherical DUSTY model, we adopt this dust density distribution as well as the chemistry from Shenoy et al. (2016) - a 50/50 mixture of astronomical silicates from Draine \& Lee (1984) and metallic iron from Harwit et al. (2001). We assume the grain radii follow an MRN size distribution $n(a) \propto a^{-3.5}$ (Mathis et al. 1977) with $a_{\min }=0.005 \mu \mathrm{m}$ and $a_{\max }=0.5 \mu \mathrm{m}$. With an effective temperature of $3490 \mathrm{~K}$ (Wittkowski et al. 2012) and an assumed dust condensation temperature of $1000 \mathrm{~K}$, DUSTY generates the model SED shown at the top of Figure 4 in red. While the dust condensation temperature can be modeled as a free parameter in DUSTY (Beasor \& Davies 2016 varied $T_{\text {in }}$ in their models in the range of $500-1200 \mathrm{~K}$ ), we assume a constant temperature of $1000 \mathrm{~K}$ to both reduce the dimensionality of our model sets and for consistency with previous work on RSG modeling in Gordon et al. (2018) and see, for example, Groenewegen (2012).

Shenoy et al. (2015) found that the SW Clump was optically thick to scattering but also highly polarized with a fractional polarization of at least $30 \%$. Since optically thick scattering tends to reduce the net polarization due to multiple scatters, the SW Clump must be relatively close to the plane of the sky and the grains must have an albedo $\omega \lesssim 0.4$ to achieve the measured level of polarization. Rather than model the clump separately in scattered light and emitted light, we use the "slab" mode in DUSTY for the SW Clump, which reproduces the scattered (reflected) and thermal emission from a central source on some planar geometry. Figure 5 illustrates the geometry of our experimental setup. The actual 3D morphology of the SW Clump is unknown, so the use of a simple slab is clearly an approximation. A spherical geometry for the clump is likely more consistent with the polarimetry; however, DUSTY cannot model this case.

To reproduce the SW Clump emission, we generate a grid of models varying the optical depths of the SW Clump material at $8.9 \mu \mathrm{m}\left(0.01<\tau_{8.9}<5\right)$. Since the SW Clump is located within a circumstellar nebula of dusty material, the radiation incident on the clump will include light partially extinguished from the star as well as radiation from hot dust between the star and the clump. Examination of the SED indicates that the bulk of the hot dust emission interior to the SW clump is emitted 


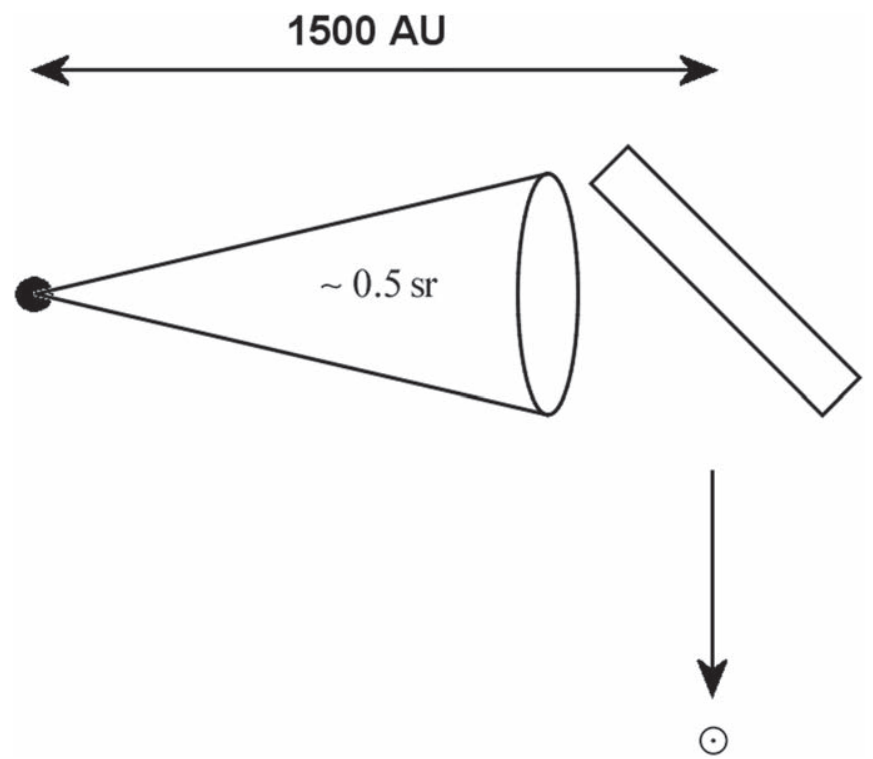

Figure 5. Geometry of the central star and the SW Clump modeled as a slab in the DUSTY code.

between 1 and $5 \mu \mathrm{m}$. Therefore, we approximate the central source seen from the clump as a blackbody with effective temperature between 1000 and $2000 \mathrm{~K}$, while maintaining the total bolometric flux. A blackbody in this temperature range would roughly peak between 2 and $5 \mu \mathrm{m}$. Since there is no spatial information on the dust emission close to the star producing the bulk of the $2-5 \mu \mathrm{m}$ emission, we did not use DUSTY to model a compact shell with an outer edge at the projected distance of the SW Clump, which would have required an unrealistic density profile.

To select a best-fitting model, we evaluate a reduced $\chi^{2}$ measurement of the observed SW Clump photometry and the DUSTY output spectrum. Unlike for the spherical case, we scale the slab DUSTY model SEDs by the solid angle subtended relative to the central star, which for our elliptical aperture is $\sim 0.5$ sr. The best-fitting model is shown in Figure 4 with the DUSTY input/output parameters summarized in Table 2. Our model fitting demonstrates that an optical depth around unity at $8.9 \mu \mathrm{m}$ is required for the slab to emit the observed flux in the mid-IR. This shows that the SW Clump is optically thick to both scattering and emission, which means we can only observe the "surface" of the clump along the light of sight. Thus, only a lower limit to the mass of the clump can be derived. The goodness-of-fit does not weight the HST $(0.4-1 \mu \mathrm{m})$ photometry here as we instead focus on the scattered and thermal emission present in the LMIRCam and NOMIC images for this work.

The luminosity of the SW Clump relative to the SED of VY CMa itself serves as an independent check on the aperture area we derived from the $2 \mu \mathrm{m}$ LMIRCam. The bolometric flux of the clump, estimated by integrating the model curve from $0.3 \mu \mathrm{m}$ through $1 \mathrm{~mm}$, is about $3 \%$ of the total luminosity of VY CMa. Our clump aperture subtends a solid angle of $\sim 0.5$ sr relative to the star, which is $\sim 4 \%$ of the full sphere. Thus, our aperture area is consistent with the observed photometry.

We note here two of our greatest uncertainties in constraining the DUSTY models: the SED of circumstellar material between the star and the SW Clump and the geometry of the
SW Clump. As discussed above, the SW Clump is not illuminated by the $3490 \mathrm{~K}$ photosphere from VY CMa, but rather a combination of attenuated light from the central star and emission from hot dusty material between the star and the clump. We have provided as input to the DUSTY slab a simple blackbody with $T=1600 \mathrm{~K}$ to approximate this incident SED, but the actual SED incident on the slab will certainly be more complicated. We note that a few hundred degree variation in the input blackbody temperature does not significantly alter the shape of the model SEDs from $5 \mu \mathrm{m}$ out to longer wavelengths.

The actual extent of the SW Clump is not fully resolved in the NOMIC images. The photometric aperture was defined from the LMIRCam $K_{s}$ image for consistency with Shenoy et al. (2013), but as we see in Figures 1 and 2, the shape of the SW Clump in scattered emission at $2 \mu \mathrm{m}$ is not the same as in thermal emission at $8.9 \mu \mathrm{m}$. Additionally, DUSTY assumes isotropic scattering from dust grains without consideration of a dependence of the scattering efficiency on scattering angle. This may in part explain the different spectral shape observed from 1 to $5 \mu \mathrm{m}$ in Figure 4 in comparison to the model. Finally, as discussed above, the $3 \mathrm{D}$ morphology of the clump is unknown. The assumptions made for the geometry lead to uncertainty in the solid angle subtended by the SW Clump relative to the central star; though, as described above, the fraction of the total sphere subtended by our aperture is consistent with the fraction of flux in the SW Clump relative to VY CMa's SED.

The data points for the SW Clump at wavelengths shorter than $2.2 \mu \mathrm{m}$ lie significantly above the model in Figure 4 . It is possible that light directly from the photosphere of VY CMa is irradiating some dust along the line of sight, increasing the scattered flux. This radiation source is not in our model, since we are only interested in the scattered light from 2 to $5 \mu \mathrm{m}$. Also, dust in front of the SW Clump that is optically thin at wavelengths longer than $2.2 \mu \mathrm{m}$, but scatter extra flux into the beam at shorter wavelengths, could also contribute to the discrepancy.

\subsection{Scattered versus Thermal Emission}

Shenoy et al. (2013) used the BHMIE code (Bohren \& Huffman 1983) to calculate the extinction and scattering efficiencies of dust grains in the SW Clump using Mie theory to determine the fractional contribution of scattering and thermal emission in the SED. At $5 \mu \mathrm{m}$, Shenoy et al. (2013) estimates that $\sim 75 \%$ of the flux in the SW Clump is due to scattered light from VY CMa. We can make a similar calculation since DUSTY also separates the scattered and thermal components of the SEDs, shown in Figure 4 with dotted and dashed lines, respectively. We derive a fractional contribution from scattering at $5 \mu \mathrm{m}$ of $92 \%$. At wavelengths longer than $10 \mu \mathrm{m}$, the emission is purely thermal.

In Table 2, we present several distinct temperature measurements. DUSTY provides estimates on the dust temperature as a function of optical depth through the slab. For the surface of the slab facing the star, the dust temperature $T_{\mathrm{d}}$ is $207 \mathrm{~K}$. As an independent check on consistency, we can roughly measure the dust temperature directly from the fluxcalibrated NOMIC images. The analogous quantity to the DUSTY temperature at the slab's surface would be an observed 
Table 2

DUSTY Model Parameters and Observed Temperatures (K)

\begin{tabular}{|c|c|c|c|c|c|c|c|c|}
\hline \multicolumn{3}{|c|}{ Inputs } & \multicolumn{3}{|c|}{ Outputs } & \multicolumn{3}{|c|}{ Observed } \\
\hline Model & $T_{\text {eff }^{\mathrm{a}}}$ & $\tau_{8.9}$ & $\overline{f_{\mathrm{sc}, 5 \mu \mathrm{m}}}$ & $T_{\mathrm{d}}^{\mathrm{c}}$ & $T_{\text {color }}^{\mathrm{d}}$ & $\overline{T_{\mathrm{BB}}^{\mathrm{e}}}$ & $T_{\text {bright }}{ }^{\mathrm{f}}$ & $T_{\text {color }}^{\mathrm{g}}$ \\
\hline slab & 1600 & 1.03 & $92 \%$ & 207 & 277 & 165 & 205 & 275 \\
\hline
\end{tabular}

Notes.

a Effective temperature of the input blackbody to model a "pseudo-photosphere" interior to the SW Clump.

${ }^{\mathrm{b}}$ Fractional contribution of scattered light to total SED flux at $5 \mu \mathrm{m}$ (M-band).

${ }^{c}$ Dust temperature in the SW Clump measured from the model at the slab boundary facing the star. See Section 3.3 for a discussion of the various temperature quantities.

${ }^{\mathrm{d}}$ Color temperature from the model SED calculated from a ratio of the 8.9 and $11.9 \mu \mathrm{m}$ flux.

e Blackbody equilibrium temperature for a 270,000 $L_{\odot}$ central source (Wittkowski et al. 2012) at the distance of the SW Clump.

${ }^{\mathrm{f}}$ Brightness temperature for the measured flux in the SW Clump.

${ }^{\mathrm{g}}$ Color temperature in the SW Clump aperture calculated from the 8.9 and $11.9 \mu \mathrm{m}$ images.

brightness temperature in the IR, calculated for a blackbody as:

$$
T_{\text {bright }}=\frac{h c}{k \lambda} \ln ^{-1}\left(1+\frac{2 h c^{2}}{I_{\lambda} \lambda^{5}}\right)
$$

For the total flux in our SW Clump aperture at $8.9 \mu \mathrm{m}$, this yields $T_{\text {bright }}=205 \mathrm{~K}$, similar to the "physical" dust temperature from DUSTY at the clump's surface.

We can also measure a color temperature from both the observed photometry and the model SED. The NOMIC 8.9 and $11.9 \mu \mathrm{m}$ filters bracket the $10 \mu \mathrm{m}$ silicate emission feature, and therefore sample the continuum emission from dust in the SW Clump. The ratio of the observed photometry yields a color temperature of $275 \mathrm{~K}$. We recover a similar measurement from the model SED of $277 \mathrm{~K}$, which is not surprising since the $\chi^{2}$-fitting performed on our grid of DUSTY models guarantees a recovered SED with a similar spectral shape to the observed photometry in the IR. Also included for comparison in Table 2 is the blackbody equilibrium temperature evaluated at a distance of $1500 \mathrm{au}$ from a source with the bolometric luminosity of VY CMa $\left(270,000 L_{\odot}\right.$; Wittkowski et al. 2012).

\subsection{Mass Estimates}

Since the optically thick $\tau_{8.9}=1.03$ DUSTY model recovers the observed total flux in the SED, we can estimate the total mass in the SW Clump from its optical depth, the grain size distribution, and the extinction efficiency. Optical depth is defined as

$$
\tau_{\lambda} \equiv \int_{a_{\min }}^{a_{\max }} Q_{\lambda} n(a) \pi a^{2} d a,
$$

where $Q_{\lambda}$ is the extinction efficiency factor, and $n(a)$ is the column density of grains with an MRN grain size distribution discussed in Section 3.2. Since our input grains to the DUSTY models are 50\% silicates and 50\% iron, our efficiency is simply the average of the efficiency functions from Draine \& Lee (1984) and Harwit et al. (2001; here, $Q \approx 0.05$ at $8.9 \mu \mathrm{m}$ ). We assume that both the extinction efficiency and the internal mass density of the grains $(\rho)$ are constant with grain size, which is reasonable since we are in the Rayleigh regime at 8-9 $\mu \mathrm{m}$, and we define the column mass density as:

$$
m\left(\mathrm{~g} \mathrm{~cm}^{-2}\right)=\rho \int_{a_{\min }}^{a_{\max }} n(a) \frac{4}{3} \pi a^{3} d a,
$$

where $\rho$ is a typical grain mass density of $3 \mathrm{~g} \mathrm{~cm}^{-3}$. We find $m=4.2 \times 10^{-4}\left(\mathrm{~g} \mathrm{~cm}^{-2}\right)$. Multiplying by the total area in the clump-2.6 $\times 10^{32} \mathrm{~cm}^{2}$ for our aperture at a distance of $1.2 \mathrm{kpc}$ (from VLBA parallax; Zhang et al. 2012) -we derive a mass of $5.4 \times 10^{-5} M_{\odot}$ in dust. Adopting a gas:dust ratio of 100:1 (for consistency with Shenoy et al. 2013), yields a total mass (gas+dust) in the SW Clump of $5.4 \times 10^{-3} M_{\odot}$. Given that the SW clump emits an amount of flux close to the fraction of flux from the star it intercepts, this mass must be considered a well constrained lower limit, given our assumptions regarding the grain population.

This result is consistent with the lower limit estimate of $M \gtrsim 5 \times 10^{-3} M_{\odot}$ from imaging polarimetry at $3.1 \mu \mathrm{m}$ by Shenoy et al. (2013). If, however, we adopt the higher gas:dust ratio of 500:1 from Decin et al. (2006) for VY CMa, our mass estimate for the SW Clump becomes $2.7 \times 10^{-2} M_{\odot}$. Compared to the typical mass-loss rate of VY CMa of $\sim 10^{-4} M_{\odot} \mathrm{yr}^{-1}$ (Danchi et al. 1994), such a large mass in a discrete feature likely represents an extreme, localized massloss event.

For comparison, O'Gorman et al. (2015) estimates a dust mass for the Clump C feature of $\sim 2 \times 10^{-4} M_{\odot}$, which they cite as a lower limit since their calculation is in the optically thin regime. With additional Band $5(178 \mathrm{GHz})$ ALMA data, Vlemmings et al. (2017) updates this dust mass to $>1.2 \times 10^{-3} M_{\odot}$. Clump C is then almost two orders of magnitude more massive than the SW Clump. Richards et al. (2014) and O'Gorman et al. (2015) also identified a second radio-bright continuum source at or near the center of the star that they call the VY component. This source, which is too close to the star for us to image, has a dust mass estimate of $\sim 3 \times 10^{-5} M_{\odot}$, which is about half of our dust mass estimate for the SW Clump. Relative to typical RSG mass-loss rates, these localized episodes of dusty ejecta are all extraordinary examples of the extreme outflow activity from massive evolved stars.

\section{Conclusions}

High-resolution, subarcsecond imaging from 9 to $12 \mu \mathrm{m}$ with NOMIC has allowed us to isolate the peculiar SW Clump feature from the overall IR emission of VY CMa. The resulting SED of the clump alone is a powerful tool in characterizing the thermal properties of the clump relative to the central star. Through DUSTY modeling, we confirm that the clump is optically thick from 9 to $12 \mu \mathrm{m}$ and has a brightness 
temperature of $\sim 200 \mathrm{~K}$. With a firm lower limit to the dust mass of $5.4 \times 10^{-5} M_{\odot}$, the SW Clump is comparable in mass to the radio-bright Clump $\mathrm{C}$ and "VY" component identified in Richards et al. (2014) and O'Gorman et al. (2015).

At a distance of $\sim 1500 \mathrm{au}$, the SW Clump represents a recent mass-loss event from VY CMa. If we assume a value for the velocity of $25 \mathrm{~km} \mathrm{~s}^{-1}$, typical for red supergiants, then the clump would have been ejected $\lesssim 300$ yr ago.

Finally, we note that our models and estimates on thermal emission from the dust in the SW Clump are not inconsistent with the nondetection at ALMA, but they do put strong constraints on the ALMA results. The SED models predict submillimeter fluxes at or below the $3 \sigma$ ALMA detection limits, but slightly above at $1.7 \mathrm{~mm}$ using our SW Clump aperture. Our models are not at all constrained beyond the $11.9 \mu \mathrm{m}$ NOMIC photometry. Therefore, high-resolution imaging of the SW Clump in the $\sim 20-100 \mu \mathrm{m}$ regime is required to characterize fully the thermal emission from this fascinating mass-loss event.

M.G. acknowledges support from the University of Minnesota Graduate School's Doctoral Dissertation Fellowship.

Facilities: LBT (LMIRCam, NOMIC), HST (WFPC2).

Software: Astropy (Astropy Collaboration et al. 2013), DUSTY (Ivezic et al. 1997).

\section{ORCID iDs}

Michael S. Gordon (iD https://orcid.org/0000-0002-1913-2682

Terry J. Jones (iD https://orcid.org/0000-0002-8716-6980 Roberta M. Humphreys (i) https://orcid.org/0000-00031720-9807

Steve Ertel (i) https://orcid.org/0000-0002-2314-7289

Jordan Stone (iD https://orcid.org/0000-0003-0454-3718

\section{References}

Astropy Collaboration, Robitaille, T. P., Tollerud, E. J., et al. 2013, A\&A, 558, A 33
Beasor, E. R., \& Davies, B. 2016, MNRAS, 463, 1269

Bohren, C. F., \& Huffman, D. R. 1983, Absorption and Scattering of Light by Small Particles (New York: Wiley)

Cardelli, J. A., Clayton, G. C., \& Mathis, J. S. 1989, ApJ, 345, 245

Danchi, W. C., Bester, M., Degiacomi, C. G., Greenhill, L. J., \& Townes, C. H. 1994, AJ, 107, 1469

De Beck, E., Vlemmings, W., Muller, S., et al. 2015, A\&A, 580, A36

Decin, L., Hony, S., de Koter, A., et al. 2006, A\&A, 456, 549

Decin, L., Richards, A. M. S., Millar, T. J., et al. 2016, A\&A, 592, A76

Draine, B. T., \& Lee, H. M. 1984, ApJ, 285, 89

Gordon, M. S., Humphreys, R. M., Jones, T. J., et al. 2018, AJ, 155, 212

Groenewegen, M. A. T. 2012, A\&A, 540, A32

Groenewegen, M. A. T., Waelkens, C., Barlow, M. J., et al. 2011, A\&A, 526, A162

Harwit, M., Malfait, K., Decin, L., et al. 2001, ApJ, 557, 844

Hinz, P. M., Defrère, D., Skemer, A., et al. 2016, Proc. SPIE, 9907, 990704

Hoffmann, W. F., Hinz, P. M., Defrère, D., et al. 2014, Proc. SPIE, 9147, 914710

Humphreys, R. M., Davidson, K., Ruch, G., \& Wallerstein, G. 2005, AJ, 129,492

Humphreys, R. M., Helton, L. A., \& Jones, T. J. 2007, AJ, 133, 2716

Ivezic, Z., Groenewegen, M. A. T., Men'shchikov, A., \& Szczerba, R. 1997, MNRAS, 291, 121

Kamiński, T., Gottlieb, C. A., Young, K. H., Menten, K. M., \& Patel, N. A. 2013, ApJS, 209, 38

Mathis, J. S., Rumpl, W., \& Nordsieck, K. H. 1977, ApJ, 217, 425

O'Gorman, E., Vlemmings, W., Richards, A. M. S., et al. 2015, A\&A, 573, L1

Packham, C., Jones, T. J., Warner, C., et al. 2012, Proc. SPIE, 8446, 84463R

Richards, A. M. S., Impellizzeri, C. M. V., Humphreys, E. M., et al. 2014, A\&A, 572, L9

Shenoy, D., Humphreys, R. M., Jones, T. J., et al. 2016, AJ, 151, 51

Shenoy, D. P., Jones, T. J., Humphreys, R. M., et al. 2013, AJ, 146, 90

Shenoy, D. P., Jones, T. J., Packham, C., \& Lopez-Rodriguez, E. 2015, AJ, 150,15

Skemer, A. J., \& Close, L. M. 2011, ApJ, 730, 53

Skrutskie, M. F., Jones, T., Hinz, P., et al. 2010, Proc. SPIE, 7735, 77353H

Smith, N., Humphreys, R. M., Davidson, K., et al. 2001, AJ, 121, 1111

Vlemmings, W. H. T., Khouri, T., Martí-Vidal, I., et al. 2017, A\&A, 603, A92

Wittkowski, M., Hauschildt, P. H., Arroyo-Torres, B., \& Marcaide, J. M. 2012, A\&A, 540, L12

Zhang, B., Reid, M. J., Menten, K. M., \& Zheng, X. W. 2012, ApJ, 744, 23 